\section{Avaliação da acurácia de testes laboratoriais para detecção de amostras de Klebsiella pneumoniae produtora de betalactamase de espectro estendido}

\author{
Comparison of different methods for detection of Klebsiella pneumoniae isolates producers of extended \\ spectrum beta-lactamase
}

\author{
Andrea dos Santos Pereira ${ }^{1}$ \\ José Rodrigues do Carmo Filho ${ }^{2}$ \\ Maria Cristina Bronharo Tognim ${ }^{3}$ \\ Helio Silva Sader ${ }^{4}$
}

\section{unitermos resumo}

ESBL

Teste de adição de ácido

clavulânico

Etest para detecção de

betalactamase de espectro

estendido
Bactérias produtoras de betalactamase de espectro estendido (ESBL) representam um dos mais importantes problemas de resistência bacteriana nos hospitais brasileiros. A necessidade da implementação de testes que apresentem alta acurácia e baixo custo é de suma importância devido à dificuldade na detecção de ESBL. O objetivo do presente estudo foi avaliar a acurácia dos testes de adição de ácido clavulânico comercializados pela Oxoid ${ }^{\circledR}$ (Basingstoke, Inglaterra) e do Etest ESBL Screen ${ }^{\circledR}$ (AB Biodisk, Solna, Suécia) para detecção de K. pneumoniae produtoras de ESBL. Objetivamos também avaliar comparativamente a sensibilidade e a especificidade dos substratos betalactâmicos utilizados nestas metodologias. Foram avaliadas 134 amostras de K. pneumoniae isoladas em hemocultura de pacientes internados no Hospital São Paulo no período de julho de 1996 a julho de 2001. As amostras foram avaliadas quanto à produção de ESBL pelo teste de triagem preconizado pelo National Committee for Clinical Laboratory Standards (NCCLS), adição de ácido clavulânico (Oxoid ${ }^{\circledR}$ ) e Etest ESBL Screen ${ }^{\circledR}$. Foram consideradas produtoras de ESBL (padrão-ouro) amostras que apresentaram teste de triagem positivo e pelo menos um dos dois testes avaliados também positivo. O teste de adição de ácido clavulânico (Oxoid ${ }^{\circledR}$ ) apresentou 100\% de sensibilidade e 100\% de especificidade, e os substratos que apresentaram melhor desempenho neste teste foram cefotaxima e cefpodoxima, ambos com $100 \%$ de sensibilidade e especificidade. O Etest ESBL Screen ${ }^{\circledR}$ apresentou $96 \%$ de sensibilidade e $100 \%$ de especificidade, sendo que a cefotaxima mostrou novamente melhor desempenho, com $92,5 \%$ de sensibilidade e $100 \%$ de especificidade. 0 teste de adição de ácido clavulânico $\left(\mathrm{Oxoid}^{\circledR}\right)$ apresentou excelente desempenho e pode ser facilmente implementado na rotina laboratorial por ser de alta acurácia e de fáceis realização e interpretação.

\section{abstract}

Bacterial strains producing extended spectrum beta-lactamases (ESBL) represent a common resistance problem among Brazilian hospitals. Due to the difficulty of ESBL detection in the clinical laboratory, these bacterial isolates require a reproducible, efficient, and low cost detection method.

The aim of the present study was to evaluate the efficacy of detection of K. pneumoniae ESBL isolates by two methods: the Etest ESBL strip and the inhibitor potentiated disk diffusion test with clavulanic acid (clavulanate-potentiation test). The sensitivity and the specificity of beta-lactam agents against these isolates were also evaluated. The experiments were performed on a total of $134 \mathrm{~K}$. pneumoniae isolates recovered from blood specimens in our institution from July 1996 to July 2001. The samples were tested for ESBL production by the NCCLS screen test, clavulanatepotentiation test and Etest ESBL strip. Isolates presenting positive results for the screen test and for at least one of the evaluated tests were considered ESBL producers (gold standard). The results of this study yielded a 100\% specificity and sensitivity for the clavulanate-potentiation test, and the

best indicators of ESBL production were cefotaxime and cefpodoxime. The Etest ESBL strip also turned out to be a very sensitive (96\%) and specific (100\%) method, being cefotaxime the most efficient substrate. According to the results of this investigation, the clavulanate potentiation disk diffusion test displayed an excellent performance and can be easily implemented in routine clinical laboratories as a practical, reliable, and accurate method.

key words

\section{ESBL}

Clavulanate potentiation

disk diffusion

Etest ESBL Screen@
1. Aluna de mestrado da disciplina de Doenças Infecciosas e Parasitárias da Universidade Federal de São Paulo (Unifesp).

2. Aluno de doutorado da disciplina de Doenças Infecciosas e Parasitárías da Unifesp.

3. Aluna de doutorado da disciplina de Doenças Infecciosas e Parasitárias da Unifesp e docente da

Universidade Estadual de Maringá. 4. Professor da disciplina de Doenças Infecciosas e Parasitárias da Unifesp e diretor do Laboratório Especial de Microbiologia Clínica/Unifesp.

Realização do Laboratório Especial de Microbiologia Clínica da disciplina de Doenças Infecciosas e Parasitárias da Escola Paulista de Medicina (Unifesp/EPM). 


\section{Introdução}

O principal mecanismo de resistência das bactérias gram-negativas aos betalactâmicos é decorrente da produção de betalactamases, que são enzimas que catalisam a hidrólise do anel betalactâmico, impossibilitando, assim, a sua atividade antimicrobiana (10).

Klebsiella pneumoniae é um dos bacilos gram-negativos mais freqüentemente isolados como causa de infecções hospitalares (28), chegando a ser responsável por até $29 \%$ das infecções de um hospital (12). As infecções causadas por $K$. pneumoniae estão associadas com alta morbimortalidade (11).

Amostras clínicas de $K$. pneumoniae apresentam resistência intrínseca a ampicilina e carbenicilina. Isto se deve à produção de betalactamases TEM-1 e SHV-1 (11). Estas betalactamases foram reportadas pela primeira vez em 1960 em amostras de Escherichia coli e Salmonella paratyphi logo após a introdução da ampicilina como tratamento de infecções causadas por estes microrganismos. Desta maneira, desde a década de 1960, ocorreu a disseminação da betalactamase plasmidial TEM-1 por outras espécies de bactérias gram-negativas, entre elas a K. pneumoniae. Esta espécie também produz a enzima SHV-1 em baixos níveis, o que proporciona resistência a ampicilina, amoxicilina, carbenicilina, ticarcilina e cefalosporinas de primeira geração (10).

Pelo uso extensivo de antimicrobianos betalactâmicos de amplo espectro na década de 1980, microrganismos que possuíam betalactamases mediadas pelo gene TEM desenvolveram resistência a cefalosporinas de amplo espectro e monobactâmicos $(11,14)$. Este desenvolvimento de resistência se deve a mutações nos genes bla $a_{\mathrm{TEM}-1}$ e bla $a_{\mathrm{SHV}-1}$, que resultam na substituição de aminoácidos alterando o substrato específico das enzimas. Desde então, inúmeros relatos de surtos envolvendo K. pneumoniae produtoras de betalactamases de espectro estendido (ESBL) têm sido reportados $(7,23)$. Os plasmídios que carregam os genes que codificam a produção de ESBL geralmente contêm genes de resistência a outros antimicrobianos, como aminoglicosídeos, sulfonamidas, tetraciclinas e cloranfenicol.

A mudança no perfil de sensibilidade aos antimicrobianos de amostras clínicas de K. pneumoniae e relatos cada vez mais freqüentes de surtos hospitalares causados por estes agentes justificam a monitoração deste patógeno no ambiente hospitalar.

A prevalência de amostras de $K$. pneumoniae produtoras de ESBL varia entre diferentes regiões, sendo relatada como 9,6\% na França (25), 14\% no Reino Unido (9), 24\% na Grécia (26) e 9\% na Hungria (13). Estudo multicêntrico envolvendo hospitais em três diferentes cidades brasileiras demonstrou que a $K$. pneumoniae foi a causa de $9,7 \%$ das infecções de corrente sangüínea, $11 \%$ das do trato respiratório inferior, $7 \%$ das de pele e mucosas, feridas, e também por $11,7 \%$ das ocorridas nas UTIs. Destas, mais de $50 \%$ eram produtoras de betalactamase (22). Outras infecções como endocardite e meningite também podem ser causadas por este agente $(11,18)$.

Atualmente as ESBLs representam o maior grupo de betalactamases estudado mundialmente e têm sido motivo de extensivas investigações microbiológicas, bioquímicas, genéticas e epidemiológicas $(1,2,6,20,21)$.

As ESBLs têm mostrado variável expressão fenotípica. Enquanto uma ESBL pode ter maior capacidade para hidrolisar drogas como a ceftriaxona ou a cefotaxima, outra pode ter maior capacidade para hidrolisar a ceftazidima ou aztreonam. A detecção laboratorial de cepas produtoras de ESBL é muito desafiadora, pois são necessários múltiplos substratos de detecção específicos (1).

A falta de um teste com boa acurácia para a detecção de amostras produtoras de ESBL tem facilitado a disseminação hospitalar desses microrganismos. Essa dificuldade pode prolongar a permanência do paciente no hospital ou mesmo aumentar a morbimortalidade, uma vez que os pacientes podem não ser tratados adequadamente (3).

De modo geral, os métodos de triagem se baseiam no padrão de sensibilidade das amostras. Testes especiais têm sido designados especificamente para a detecção e/ou confirmação da produção de ESBL. Entre estes testes estão o teste de disco-difusão utilizando pontos de corte (breakpoints) indicativos da produção de ESBL estabelecidos pelo National Committee for Clinical Laboratory Standards (NCCLS), o teste de adição de ácido clavulânico Oxoid ${ }^{\circledR}$ (Basingstoke, Inglaterra) e o Etest ESBL Screen ${ }^{\circledR}$ (AB Biodisk, Solna, Suécia). Alguns relatos mostram que o ácido clavulânico é um inibidor mais potente do que sulbactam para enzimas TEM e SHV com espectro ampliado (18). Desta maneira, o ácido clavulânico é o composto mais utilizado nestes testes.

O objetivo do presente estudo foi avaliar comparativamente a acurácia dos testes de adição de ácido clavulânico comercializados pela Oxoid ${ }^{\circledR}$ (Basingstoke, Inglaterra) e do Etest ESBL Screen ${ }^{\circledR}$ (AB Biodisk, Solna, Suécia) para detecção de amostras de $K$. pneumoniae produtoras de ESBL. Objetivamos também avaliar comparativamente a sensibilidade e a especificidade dos substratos betalactâmicos utilizados nestas metodologias. 


\section{Materiais e métodos}

Foram incluídas no estudo 134 amostras de K. pneumoniae isoladas de corrente sangüínea e coletadas consecutivamente, sendo obtida uma única amostra por paciente. As amostras foram obtidas de pacientes internados no Hospital São Paulo da Escola Paulista de Medicina da Universidade Federal de São Paulo (EPM/Unifesp) no período de julho de 1996 a julho de 2001. Estas cepas foram isoladas e identificadas no Laboratório Central do Hospital São Paulo (Unifesp/EPM) e armazenadas a $-20^{\circ} \mathrm{C}$ no banco de microrganismos do Laboratório Especial de Microbiologia Clínica (LEMC). A identificação em gênero e espécie foi repetida no LEMC, utilizando-se provas bioquímicas convencionais (5).

Para a realização dos testes de detecção da produção de ESBL as amostras foram retiradas do banco de microrganismos e subcultivadas por duas vezes em MuellerHinton (Oxoid, Basingstoke, Inglaterra) suplementado com $5 \%$ de sangue de carneiro e ágar MacConkey.

Para cada amostra foi preparada uma suspensão bacteriana em caldo Mueller-Hinton (MHB, Oxoid, Basingstoke), com turbidez correspondente a 0,5 da escala de McFarland, utilizando-se o nefelômetro Abbot A-just ${ }^{\circledR}$ (Abbot, Deerfield, EUA). Após homogeneização desta suspensão foi realizada a semeadura em uma placa $(15 \times 150 \mathrm{~mm})$ de ágar MuellerHinton (Oxoid, Basingstoke, Inglaterra) sendo que os discos foram dispensados nas placas até 15 minutos após a semeadura, seguindo as normas preconizadas pelo NCCLS (15). A seguir, os discos da marca Oxoid ${ }^{\circledR}$ de aztreonam (30 $\mu$ g),

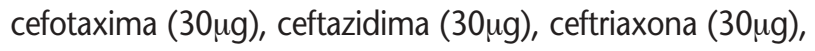
cefpodoxima $(2 \mu \mathrm{g})$, ceftazidima com ácido clavulânico

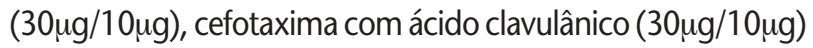
e cefpodoxima com ácido clavulânico $(10 \mu \mathrm{g} / 1 \mu \mathrm{g})$ foram dispensados na superfície do ágar. As placas foram incubadas por 18 a 24 horas, à temperatura de $35^{\circ} \mathrm{C}$, sendo os halos de inibição aferidos após este período.

Foram consideradas produtoras de ESBL (padrão-ouro) as cepas positivas para o teste de triagem estabelecido pelo NCCLS e que também fossem positivas para um dos testes avaliados: teste de adição de ácido clavulânico ou Etest ESBL Screen ${ }^{\circledR}$. A positividade para pelo menos um dos substratos testados indica que o teste é positivo, sendo que este critério foi utilizado para todos os testes analisados neste estudo.

A leitura do teste de disco-difusão para os substratos cefotaxima, ceftriaxona, ceftazidima, cefpodoxima e aztreonam foi analisada de acordo com os breakpoints utilizados para triagem de cepas de K. pneumoniae e Escherichia coli produtoras de ESBL estabelecidos pelo NCCLS $(15,16)$ (Tabela 1). O teste de triagem foi considerado positivo quando a amostra apresentava halo de inibição menor que estes breakpoints para pelo menos um substrato.

O teste de adição de ácido clavulânico foi realizado como descrito acima e utilizando-se discos de ceftazidima/ácido clavulânico $(30 \mu \mathrm{g} / 10 \mu \mathrm{g})$, cefotaxima/ácido clavulânico $(30 \mu \mathrm{g} / 10 \mu \mathrm{g})$ e cefpodoxima/ácido clavulânico $(10 \mu \mathrm{g} / 1 \mu \mathrm{g})$ disponíveis comercialmente (Oxoid, Basingstoke, Inglaterra). O teste foi considerado positivo quando os diâmetros dos halos dos antimicrobianos associados ao ácido clavulânico foram pelo menos $5 \mathrm{~mm}$ mais elevados que os diâmetros dos antimicrobianos sem o ácido clavulânico. O teste de adição de ácido clavulânico foi considerado positivo quando pelo menos um dos três substratos utilizados apresentou positividade neste teste.

Foram utilizadas duas fitas de Etest ${ }^{\circledR}$ (AB Biodisk, Solna, Suécia) disponíveis comercialmente para a detecção de ESBL. Uma das fitas contém concentrações crescentes de ceftazidima $(0,5 \mu \mathrm{g} / \mathrm{ml} \mathrm{a} 32 \mu \mathrm{g} / \mathrm{ml})$ em uma das extremidade e concentrações crescentes de ceftazidima $(0,064 \mu \mathrm{g} / \mathrm{ml} \mathrm{a}$ $4 \mu \mathrm{g} / \mathrm{ml})$ associadas a uma concentração fixa $(4 \mu \mathrm{g} / \mathrm{ml})$ de ácido clavulânico na outra extremidade. A outra fita utilizada contém cefotaxima em concentrações crescentes $(0,25 \mu \mathrm{g} / \mathrm{ml}$ a $16 \mu \mathrm{g} / \mathrm{ml})$ em uma das extremidades e, na outra extremidade, concentrações crescentes de cefotaxima $(0,16 \mu \mathrm{g} / \mathrm{ml}$ a $1 \mu \mathrm{g} / \mathrm{ml})$ associadas a uma concentração fixa de ácido clavulânico $(4 \mu \mathrm{g} / \mathrm{ml})$. Uma amostra foi considerada produtora de ESBL quando ocorreu

Breakpoints utilizados para triagem de cepas de Klebsiella spp. e Escherichia coli produtoras de ESBL, Tabela 1 segundo o NCCLS*

\begin{tabular}{lcc} 
Antimicrobianos & $\begin{array}{c}\text { Disco-difusão } \\
(\mathrm{mm})\end{array}$ & $\begin{array}{c}\text { MIC } \\
(\mu \mathrm{g} / \mathrm{ml})\end{array}$ \\
Cefotaxima & $\leq 27$ & $\geq 2$ \\
Ceftriaxona & $\leq 25$ & $\geq 2$ \\
Ceftazidima & $\leq 22$ & $\geq 2$ \\
Cefpodoxima & $\leq 17$ & $\geq 8$ \\
Aztreonam & $\leq 27$ & $\geq 2$ \\
\hline
\end{tabular}

*NCCLS, 2002. 
redução da concentração inibitória mínima $(\mathrm{CIM})$ de ceftazidima $\geq 3$ diluições logarítmicas na presença de ácido clavulânico em comparação com a CIM de ceftazidima sem o mesmo (16), ou seja, quando a razão entre a CIM do antimicrobiano testado e a CIM do antimicrobiano associado ao ácido clavulânico for $\geq 8(3,6)$. Em casos onde ambas as CIMs apresentaram valores abaixo dos gradientes de antimicrobianos presentes na fita, a redução foi considerada zero e o teste, negativo $(3,6)$. Por outro lado o teste foi considerado indeterminado quando ambas as MICs se encontravam acima da escala da fita, impossibilitando assim o cálculo da redução da $\operatorname{CIM}(3,6)$.

Amostras da American Type Culture Collection (ATCC), K. pneumoniae ATCC 700603 produtora de ESBL e E. coli ATCC 25922 não-produtora de ESBL, foram utilizadas como controle de qualidade para todos os testes de sensibilidade realizados neste estudo. As cepas ATCCs foram avaliadas seguindo a padronização do NCCLS e a interpretação dos resultados foi realizada utilizando os limites preconizados para cada ATCC $(15,16)$.

\section{Resultados}

De acordo com o padrão-ouro estabelecido nesse estudo, 72 amostras $(53,8 \%)$ foram classificadas como produtoras de ESBL e $62(46,2 \%)$ como não-produtoras. Este resultado representa a prevalência da produção de ESBL em amostras de K. pneumoniae isoladas de hemocultura em pacientes internados no Hospital São Paulo no período de julho de 1996 a julho de 2001.

Todas as amostras que apresentaram teste de triagem negativo de acordo com as normas estabelecidas pelo NCCLS apresentaram também resultados negativos para os demais testes avaliados no presente estudo, enfatizando assim a especificidade deste teste $(15,16)$.

Entre as 72 amostras consideradas produtoras de ESBL pelo padrão-ouro todas foram detectadas pelo teste de adição de ácido clavulânico. Porém três amostras foram consideradas indeterminadas pelo Etest ESBL Screen ${ }^{\circledR}$. Nesses três casos, as CIMs para os dois compostos (ceftazidima e ceftazidima/ácido clavulânico) se encontravam acima da escala da fita de Etest ESBL Screen ${ }^{\circledR}$, o que caracteriza o resultado do teste como indeterminado de acordo com as normas do fabricante (AB Biodisk, Solna, Suécia).

Na Tabela 2 podemos observar a sensibilidade e as especificidades de cada substrato utilizado no teste de triagem preconizado pelo NCCLS (16). Os substratos cefotaxima, cefpodoxima e ceftriaxona apresentaram sensibili- dade e especificidade de $100 \%$. A ceftazidima $(97,3 \%$ de sensibilidade) não detectou duas amostras produtoras de ESBL, e a aztreonam ( $94,7 \%$ de sensibilidade) não detectou quatro amostras. Além disso, uma amostra apresentou teste de triagem positivo somente pela aztreonam, sendo que os demais substratos e os testes confirmatórios foram negativos. Desta maneira este teste foi considerado falso positivo.

O teste de adição de ácido clavulânico da Oxoid $^{\circledR}$ (Basingstoke, Inglaterra) apresentou sensibilidade e especificidade de $100 \%$ (Tabela 3). Os substratos utilizados neste teste foram ceftazidima, cefotaxima e cefpodoxima, sendo que os dois últimos apresentaram 100\% de sensibilidade (Figura 1). A ceftazidima não detectou a produção da enzima para duas amostras consideradas ESBL-positivas para os demais testes. Podemos observar na Tabela 4 a sensibilidade e a especificidade de cada substrato analisado individualmente.

O teste Etest ESBL Screen ${ }^{\circledR}$ (AB Biodisk, Solna, Suécia) apresentou sensibilidade de $96 \%$ e especificidade de $100 \%$ (Tabela 3). O substrato que apresentou melhor desempenho neste teste foi a cefotaxima, com $92,5 \%$ de sensibilidade. Os dois substratos analisados (ceftazidima e cefotaxima) apresentaram especificidade de 100\% (Tabela 5 e Figuras 2 e 3).

A fita com ceftazidima não detectou a produção da enzima para cinco amostras, e para outras três amostras os resultados foram indeterminados. Por outro lado, a fita com cefotaxima apresentou resultado negativo para duas amostras e indeterminado para três.

Sensibilidade e especificidade dos diferentes substratos utilizados no teste de triagem preconizado pelo NCCLS para detecção de ESBL em Tabela 2134 isolados de K. pneumoniae

\begin{tabular}{lcc}
$\begin{array}{c}\text { Antimicrobiano } \\
(\%)\end{array}$ & $\begin{array}{c}\text { Sensibilidade } \\
(\%)\end{array}$ & $\begin{array}{c}\text { Especificidade } \\
\text { Ceftazidima }\end{array}$ \\
Cefotaxima & $97,3^{*}$ & 100 \\
Cefpodoxima & 100 & 100 \\
Ceftriaxona & 100 & 100 \\
Aztreonam & 100 & 100 \\
\hline
\end{tabular}

*A ceftazidima não detectou a produção de ESBL em duas amostras; **a aztreonam não detectou a produção de ESBL em quatro amostras; ***uma amostra não é produtora de ESBL, mas somente a aztreonam a classificou como possível produtora de ESBL. 


\section{Sensibilidade e especificidade de}

cada método avaliado para detecção da produção de ESBL em 134

\section{Tabela 3 isolados de K. pneumoniae}

\begin{tabular}{|c|c|c|}
\hline Método & $\begin{array}{c}\text { Sensibilidade } \\
(\%)\end{array}$ & $\begin{array}{c}\text { Especificidade } \\
(\%)\end{array}$ \\
\hline Etest ESBL Screen ${ }^{\circledR}$ & $96 *$ & 100 \\
\hline $\begin{array}{c}\text { Adição do ácido } \\
\text { clavulânico }\end{array}$ & 100 & 100 \\
\hline Triagem NCCLS & 100 & 100 \\
\hline
\end{tabular}

*Três amostras foram consideradas indeterminadas por este método.

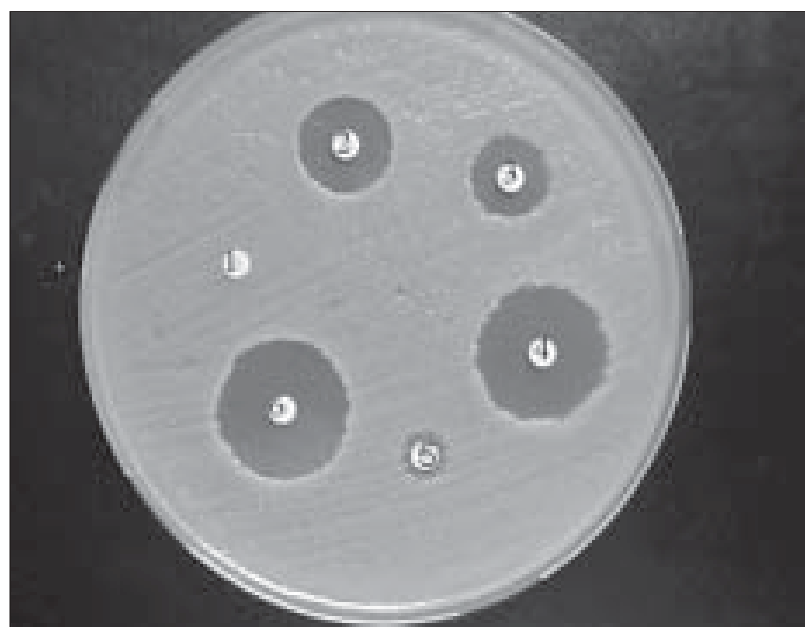

Figura 1 - Teste de adição de ácido clavulânico (Oxoid $\left.{ }^{\circledR}\right)$ positivo em amostra de K. pneumoniae. Um aumento $\geq 5 \mathrm{~mm}$ é observado nos halos dos antimicrobianos associados ao ácido clavulânico quando comparados com os antimicrobianos sem o ácido clavulânico. 1 = cefpodoxima; 2 = cefpodoxima + ácido clavulânico; 3 = ceftazidima; 4 = ceftazidima + ácido clavulânico; 5 = cefotaxima; 6 = cefotaxima + ácido clavulânico

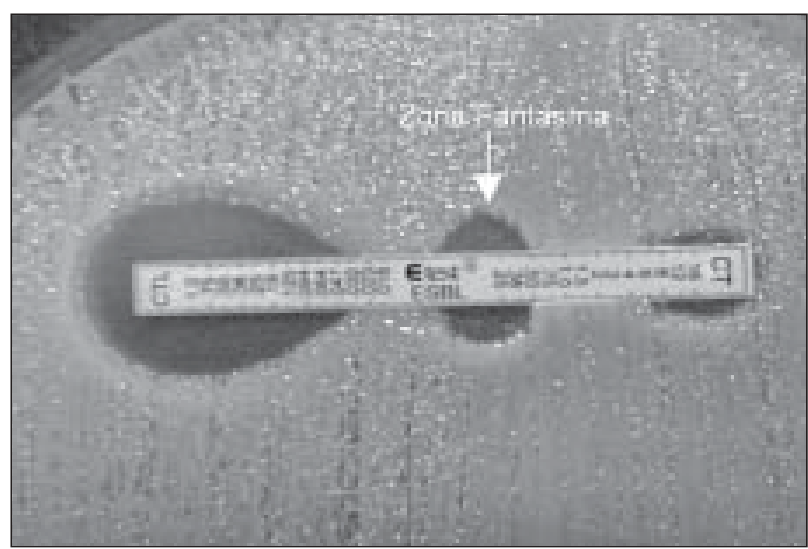

Figura 2 - Etest ESBL Screen ${ }^{\circledR}$ positivo para o substrato cefotaxima em amostra de K. pneumoniae. Além da diminuição $\geq 3$ escalas logarítmicas na CTL quando comparada com CT, a presença desta zona fantasma indica produção de ESBL devida ao sinergismo entre cefotaxima e cefotaxima/ácido clavulânico, MIC CT/CTL = 12/ 0,047 $\mathrm{g} / \mathrm{ml} . \mathrm{CT}$ = cefotaxima; CTL = cefotaxima + ácido clavulânico

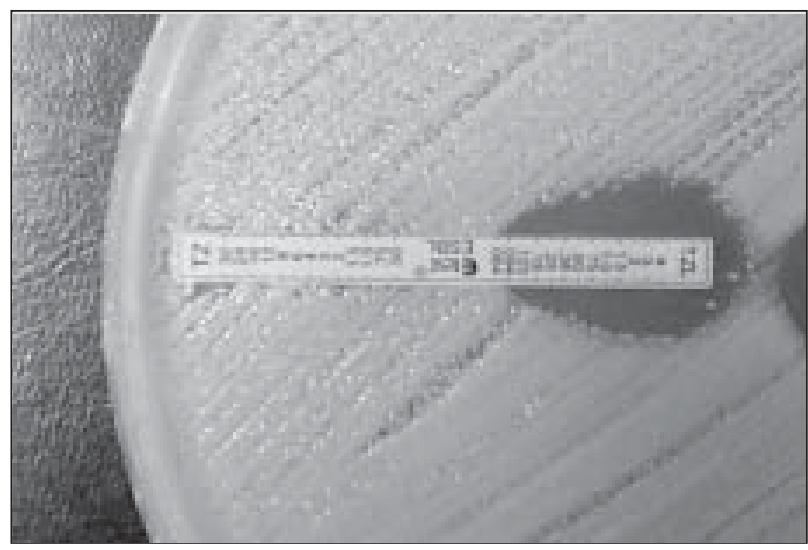

Figura 3 - Etest ESBL Screen ${ }^{\circledR}$ positivo para o substrato ceftazidima em amostra de K. pneumoniae. Observa-se a diminuição da CIM para ceftazidima/ácido clavulânico. CIM TZ/TZL $\geq 32 / 0,0125 \mathrm{\mu g} / \mathrm{ml}$. TZ = ceftazidima; TZL = ceftazidima + ácido clavulânico
*A ceftazidima não detectou a produção de ESBL para oito amostras; **a

cefotaxima não detectou a produção de ESBL para cinco amostras.

Sensibilidade e especificidade dos diferentes substratos testados pela metodologia do Etest ESBL Screen ${ }^{\circ}$ para detecção da produção de ESBL

Tabela 5 em 134 isolados de K. pneumoniae

\begin{tabular}{lcc}
\hline Antimicrobiano & $\begin{array}{c}\text { Sensibilidade } \\
(\%)\end{array}$ & $\begin{array}{c}\text { Especificidade } \\
(\%)\end{array}$ \\
Ceftazidima & $90^{*}$ & 100 \\
Cefotaxima & $92,5^{* *}$ & 100 \\
\hline
\end{tabular}

\section{Sensibilidade e especificidade dos diferentes substratos do teste de adição de ácido clavulânico da Oxoid @e para detecção da produção de ESBL} Tabela 4 em 134 isolados de K. pneumoniae

\begin{tabular}{lcc}
\hline Antimicrobiano & $\begin{array}{c}\text { Sensibilidade } \\
(\%)\end{array}$ & $\begin{array}{c}\text { Especificidade } \\
(\%)\end{array}$ \\
Ceftazidima & $97,3^{*}$ & 100 \\
Cefotaxima & 100 & 100 \\
Cefpodoxima & 100 & 100 \\
\hline
\end{tabular}

*A ceftazidima não detectou a produção de ESBL para duas das amostras. 


\section{Discussão}

A pesquisa de bactérias produtoras de ESBL é de suma importância nos laboratórios de microbiologia clínica. Sua detecção, entretanto, é dificultada pelo fato de a acurácia do teste estar relacionada ao tipo de enzima produzido $(1,8)$. A determinação de ponto isoelétrico das diferentes betalactamases poderá auxiliar na determinação da classe enzimática presente nas amostras bacterianas. A caracterização específica destas enzimas, entretanto, só poderá ser verificada mediante a realização de métodos moleculares (24).

A sensibilidade e a especificidade de cada teste dependerá da coleção de amostras utilizadas na avaliação. No presente estudo foi utilizada uma coleção de amostras altamente representativa, pois se trata de isolados de hemocultura coletados consecutivamente em um período de cinco anos.

Outra dificuldade encontrada na avaliação de testes para detecção de cepas produtoras de ESBL é o estabelecimento de um padrão-ouro, uma vez que algumas cepas expressam um grau muito baixo de resistência, ou, ainda, a produção de ESBL pode estar associada a outro mecanismo de resistência aos betalactâmicos. Desta maneira, nenhum teste fenotípico pode ser considerado padrãoouro, pois poderá apresentar tanto resultados falsos positivos quanto falsos negativos (27).

Para contornar esta situação muitos estudos utilizam uma combinação de testes como padrão-ouro (4). No presente estudo foram consideradas produtoras de ESBL as amostras que apresentaram teste de triagem positivo para qualquer substrato e que também foram positivas para pelo menos um dos dois testes que avaliam o efeito do ácido clavulânico. O teste de triagem apresenta alta sensibilidade, enquanto os testes que avaliam o efeito do ácido clavulânico apresentam alta especificidade. Assim, esta combinação representa um padrão-ouro bastante adequado.

Ambos os testes avaliados, adição de ácido clavulânico Oxoid $^{\circledR}$ (Oxoid, Basingstoke, Inglaterra) e Etest ESBL Screen ${ }^{\circledR}$ (AB Biodisk, Solna, Suécia), apresentaram resultados confiáveis. Na verdade, os testes utilizados apresentaram poucas discordâncias. Caso somente ceftazidima e aztreonam tivessem sido utilizadas no teste de triagem, duas amostras para ceftazidima e quatro para aztreonam não teriam sido adequadamente categorizadas quanto à produção de ESBL.

Os problemas encontrados com os substratos ceftazidima (97,3\% de sensibilidade) e aztreonam (94,7\% de sensibilidade e $98,6 \%$ de especificidade) devem ser decorrentes, possivelmente, da maior prevalência de enzimas do grupo SHV e CTX. Porém estudos moleculares são necessários para identificação das ESBLs mais prevalentes no Brasil $(1,20)$. Apesar da baixa especificidade relatada para cefpodoxima em alguns estudos, não encontramos nenhum resultado falso positivo com este mesmo substrato (Tabelas 2 e 3).

O teste de adição de ácido clavulânico comercializado pela Oxoid ${ }^{\circledR}$ (Basingstoke, Inglaterra) apresentou excelente desempenho com $100 \%$ de sensibilidade e $100 \%$ de especificidade. Cefotaxima e cefpodoxima apresentaram novamente desempenho melhor que a ceftazidima como substrato. É importante ressaltar que duas amostras não-detectadas pela ceftazidima neste teste foram as mesmas que apresentaram testes de triagem e Etest ESBL Screen ${ }^{\circledR}$ negativo para este mesmo substrato (Tabelas 2 e 3 ).

O Etest ESBL Screen ${ }^{\circledR}$ (AB Biodisk, Solna, Suécia) também apresentou bom desempenho, com $96 \%$ de sensibilidade e $100 \%$ de especificidade. Neste teste o substrato que apresentou melhor desempenho na detecção da produção de ESBL foi mais uma vez a cefotaxima. É importante ressaltar que, caso os testes que apresentaram CIMs acima da escala em ambas extremidades da fita fossem considerados positivos, isto é, as cepas fossem consideradas produtoras de ESBL, o Etest ESBL Screen ${ }^{\circledR}$ também teria apresentado $100 \%$ de sensibilidade. Porém estas amostras não podem ser consideradas produtoras de ESBL porque outros mecanismos de resistência que podem não levar a resistência cruzada a todas cefalosporinas, monobactâmicos e penicilinas de amplo espectro poderiam causar elevação da CIM acima das escala em ambos os lados da fita (17).

Quando comparados Etest ESBL Screen ${ }^{\circledR}$ e discos associados ao ácido clavulânico (Oxoid, Basingstoke, Inglaterra) podemos verificar que, apesar de o disco não avaliar quantitativamente a inibição da enzima, o método detectou todas as amostras produtoras de ESBL, incluindo aquelas não-detectadas pelo Etest ESBL Screen ${ }^{\circledR}$. Este dado é muito importante, uma vez que os discos podem facilmente ser adicionados ao antibiograma na rotina laboratorial sem acarretar grandes mudanças nesta rotina. Além disso, trata-se de um método de fáceis realização e interpretação e menor custo.

Trabalhos anteriores à comercialização dos discos de ácido clavulânico mostraram baixa especificidade deste teste quando discos confeccionados in house eram utiliza- 
dos (20). Por ser uma substância altamente instável, o cuidado com a pronta realização do teste após a adição do ácido clavulânico e o correto armazenamento desta substância são fatores determinantes para um bom desempenho do teste.
Os resultados aqui apresentados são de extrema importância, uma vez que a detecção acurada e rápida de amostras produtoras de ESBL em espécimes clínicos permite uma melhor escolha terapêutica e dificulta, assim, a disseminação deste tipo de resistência.

\section{Referências}

1. Bush, K.; Jacob, G .A.\& Medeiros, A .A . A functional classification scheme for $\beta$-lactamases and its correlation with molecular structure. Antimicrob.Agents Chemother., 39:1211-33,1995.

2. Casellas, J.M.; Blanco, M.G .\& Pinto, M.E. The sleeping giant. A ntimicro bial resistance. Infect. Dis. Clin. North Am., 8: 2945, 1994.

3. Cormican, M.G.; Marshall, S.A. \& Jones, R.N . Detection of extended-spectrum $\beta$-lactamases (ESBL)-producing strains by Etest ESBL screen. J. Clin. M icrobiol., 34: 1880-4, 1996.

4. D eshpande, L.M. et al. Accuracy of both microdilution and Etest methods for detecting chloramphenicol acetyl transferase mediated resistance in Streptococcus pneumoniae: Geographic variations in the prevalence of resistance in The Sentry A ntimicrobial Surveillance Program (1999). Diagn. M icrobiol. Infect. Dis., 39: 267-9, 2001.

5. Farmer,J.J. Enterobacteriaceae: Introduction and identification. In: Murray, P.R. et al. M anual of Clinical M icrobiology, 7.ed. ASM Press W ashington, DC: 1999. p. 442-58.

6. Gales, A.C . et al. A ntimicrobial susceptibility of Klebsiella pneumoniae producing extended-spectrum beta-lactamase (ESBL) isolated in hospital in Brazil. Braz. J. Infect. Dis., 1:196-203, 1997.

7.G niadkowski, M.et al. 0 utbreak of ceftazidime-resistant Klebsiella pneumoniae in a pediatric Hospital in W arsaw, Poland: Clonal spread of the TEM-47 extended-spectrum $\beta$-lactamase (ESBL)-producing strain and transfer of a plasmid carrying the SH V-5-like ESBL-encoding gene. Antimicrob. Agents Chemother., 42: 3079-85, 1998.

8. Jaco by, G A. et al. Co mparative in vitro activities of carbapenem L-749, 345 and other antimicrobials against multiresistant Gram-negative clinical pathogens. Antimicrob. Agents Chemother., 41: 1830-1, 1997.

9. Lin, P.Y.F. et al. Survey of the prevalence of betalactamases amongst $1000 \mathrm{Gram}$-negative bacilli isolated consecutively at the Royal London Hospital. J.Antimicrob. Chemother., 30 : $429,1992$.

10. Livermore, D.M. $\beta$-lactamases in laboratory and clinical resistance. Clin. Microbiology Rev., 8: 557-84, 1995.

11. Meyer, K.S. et al. N osocomial o utbreak of Klebsiella pneumoniae infection resistant to late-generation cephalo sporins. Ann. Intern. Med., 119: 353-8, 1993.

12. Myerowitz,J.L.; M edeiros,A .D.\& 0 'Brien,T.F.Recent experience with bacillemia due $\mathrm{G}$ ram-negative organisms. J. Infect. Dis., 124:239-46, 1971.

13. $\mathrm{N}$ agy, E. et al. Investigation of the presence of different broadspectrum betalactamases among clinical isolates of
Enterobacteriaceae. Acta M icrobiol. Immunol. Hug., 45(3-4): 433-46, 1998.

14. $\mathrm{N}$ aumovski, L. et al. 0 utbreak of ceftazidime resistance due to novel extended-spectrum $\beta$-lactamase in isolates from cancer patients. Antimicrob. Agents Chemother., 36: 1991-6, 1992.

15. N ational Committee for Clinical Laboratory Standards (N CCLS). Approved standard M 2-A7: Performance standards for antimicrobial disk susceptibility tests. 7.ed. W ayne, PA, 2000.

16. N ational Committee for Clinical Laboratory Standards ( N C CLS). Performance standards for antimicrobial susceptibility testing. $8^{\text {th }}$ informational supplement table M100-S12 (M 7).W ayne, PA, 2002.

17. Pango $n, B$. et al. In vivo selection of cephamycin-resistant, porindeficient mutant of Klebsiella pneumoniae producing a TEM-3 betalactamase. J. Infect. Dis., 159: 1005-6, 1989.

18. Payne, D.J. et al. Comparative activities of clavulanic acid, sulbactam, and tazobactam against clinically important b-lactamases. Antimicrob. Agents Chemother., 38: 767-72, 1994.

19. Pragai, Z.; Kóczián, Z \& N agy, E. Characterization of the extended-spectrum $\beta$-lactamases and determination of the antibiotic susceptibilities of Klebsiella pneumoniae isolates in H ungary. J. Antimicrob. Chemother., 42: 401-3, 1998.

20. Reis, A .O .; Gales, A.C .\& Sader, H.S. Avaliação da acurácia do teste de adição clavulanato em disco para a detecção de amostras de Klebsiella pneumoniae produtoras de ESBL. J. Bras. Patol., 34: 85-93, 1998.

21. Sader, H.S. \& Jones, R.N. In vitro antimicrobial activity of cefpirome against ceftazidime-resistant isolates from two multicenter studies. Eur. J. Clin. M icrobiol. Infect. Dis., 13:675-9, 1994.

22. Sader, H.S. et al. Pathogen frequency and resistance patterns in Brazilian hospitals: summary of results from three years of the Sentry Antimicrobial Surveillance Program. Braz. J. Infect. Dis.; 5: 200-14, 2001.

23. Schooneveldt, J.M.; N immo, G.R. \& G iffard, P. D etection and characterization of extended spectrum $\beta$-lactamases in Klebsiella pneumoniae causing nosocomial infection. Pathology., 30: 164-8, 1998.

24. Steward, C.D. et al. Characterization of clinical isolates of Klebsiella pneumoniae from 19 laboratories using the $\mathrm{N}$ ational Committee for Clinical Laboratory Standards extended-spectrum $\beta$-lactamase detection methods.J. Clin. Microbiol., 39: 2864-72, 2001. 
25. Sirot, D. et al. Transferable resistance to third-generation cephalosporins in clinical isolates of $\mathrm{K}$. pneumoniae identification of CTX-1, a novel beta-lactamase.J. Antimicrob. Chemother., 20: 323-4, 1987.

26.Vatopoulos,A.C. et al. Prevalence of a transferable SH V-5 type $\beta$-lactamase in clinical isolates of Klebsiella pneumoniae and Escherichia coli in Greece. J. Antimicrob. Chemother., 16: 635-48, 1990.
27. Yamazumi, T. et al. Comparison of the Vitek gram-positive susceptibility 106 card, the MRSA Screen latex agglutination test, and mecA analysis for detecting oxacillin resistance in a geographically diverse collection of isolates of coagulase-negative staphylococci.J.Clin. M icrobiol., 39:363336, 2001.

28. Yinnon, A.M. et al. Klebsiella bacteremia: community versus nosocomial infections. Q.J. Med., 89: 933-41, 1996. 